Influence of hydrogen absorption on structural and electrical transport properties of $\mathrm{Ho}_{1-x} \mathrm{Mm}_{x} \mathrm{Co}_{2}$ alloys

G. SrinivasV. SankaranarayananS. Ramaprabhu

Citation: Journal of Applied Physics 102, 063706 (2007); doi: 10.1063/1.2757004

View online: $\mathrm{http}: / / d x . d o i . o r g / 10.1063 / 1.2757004$

View Table of Contents: http://aip.scitation.org/toc/jap/102/6

Published by the American Institute of Physics

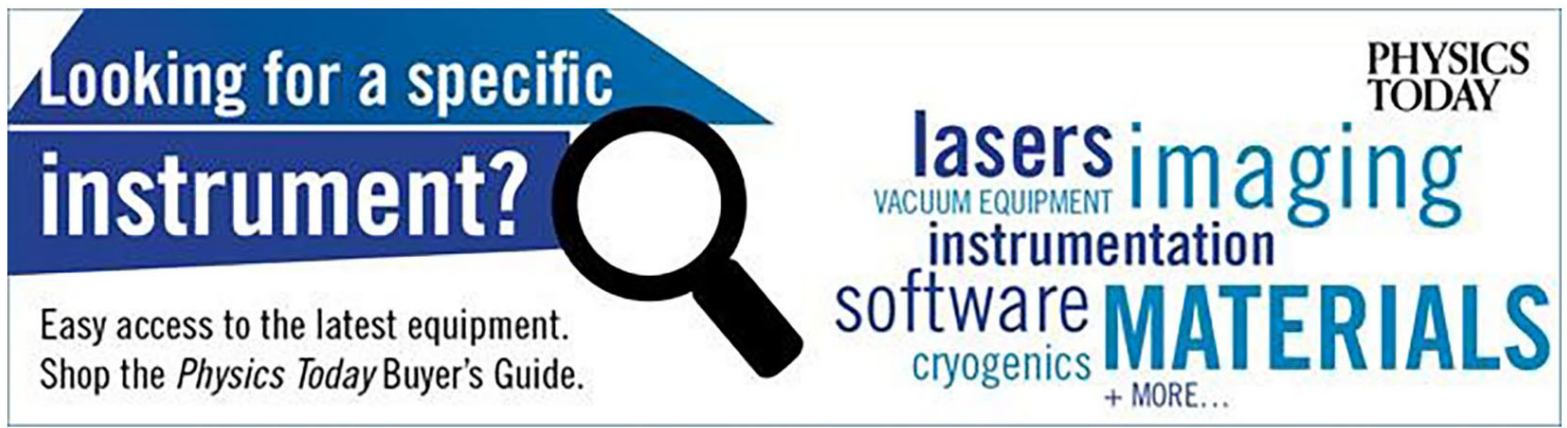




\title{
Influence of hydrogen absorption on structural and electrical transport properties of $\mathrm{Ho}_{1-x} \mathrm{Mm}_{x} \mathrm{Co}_{2}$ alloys
}

\author{
G. Srinivas \\ Low Temperature Laboratory, Department of Physics, Indian Institute of Technology, Madras, \\ Chennai 600 036, India and Alternative Energy Technology Laboratory, Department of Physics, \\ Indian Institute of Technology, Madras, Chennai 600 036, India \\ V. Sankaranarayanan \\ Low Temperature Laboratory, Department of Physics, Indian Institute of Technology, Madras, \\ Chennai 600 036, India \\ S. Ramaprabhu ${ }^{\text {a) }}$ \\ Alternative Energy Technology Laboratory, Department of Physics, Indian Institute of Technology, Madras, \\ Chennai 600 036, India
}

(Received 14 April 2007; accepted 2 June 2007; published online 19 September 2007)

\begin{abstract}
The structural and electrical transport properties of $\mathrm{Ho}_{1-x} \mathrm{Mm}_{x} \mathrm{Co}_{2}(x=0,0.1,0.2,0.3$, and 0.4 and $\mathrm{Mm}=$ mischmetal) alloys and their hydrides in the hydrogen concentration $(y)$ range of $0 \leqslant y$ $\leqslant 3.6$ have been determined through the powder x-ray diffraction (XRD) and temperature dependence of electrical resistivity $[\rho(T)]$ measurements. At room temperature, these compounds crystallize in $\mathrm{MgCu}_{2}$-type (C15) structure. The crystalline nature and lattice expansion of hydrogenated alloys have been studied using the hydrogen concentration dependence of XRD peak analysis indicating the coexistence of two hydride phases at intermediate hydrogen concentrations. The temperature dependence of the electrical resistivity of alloys has been discussed based on the conduction electron scattering and spin fluctuation scattering mechanisms. The changes in magnetic ordering and transition temperature upon $\mathrm{Mm}$ substitution and at different concentrations of hydrogen loadings have been discussed. Furthermore, the transformation from metalliclike conductivity to thermally activated conduction mechanism and different conduction regimes of temperature dependent resistivity upon increasing $\mathrm{H}$ concentration have been well described by Kondo-type and electron-electron scattering effects. () 2007 American Institute of Physics.
\end{abstract} [DOI: $10.1063 / 1.2757004]$

\section{INTRODUCTION}

Most of the Laves phase $A B_{2}$ compounds formed among rare earths $(R)$ and elements of the $d$-transition series $(M)$ form stable hydrides and, very often, hydrogen absorption leads to strong changes of the macroscopic structural, electronic, and magnetic properties of these materials due to the bonding between interstitial $\mathrm{H}$ atoms. ${ }^{1-9}$ Laves phase $R \mathrm{Co}_{2}$ and $\mathrm{LR}_{1-x} \mathrm{HR}_{x} \mathrm{Co}_{2}(\mathrm{LR}=$ light rare earth and $\mathrm{HR}=$ heavy rare earth) compounds show unusual varieties of physical properties and the results indicate that the physical properties are strongly dependent on the type of rare earth element and the lattice constant. ${ }^{10-15} \mathrm{HoCo}_{2}$ belongs to the $R \mathrm{Co}_{2}$ family, crystallizes in $\mathrm{MgCu}_{2}$-type structure, and has enhanced paramagnetic state at room temperature. It readily absorbs large amount of hydrogen, about 3.5-4 H/f.u. and forms stable hydrides. The hydrogen absorption in $\mathrm{HoCo}_{2}$ leads to the weakening of magnetic interactions. ${ }^{8,16,17}$ At higher hydrogen concentrations, the system goes from metallic state to semiconducting- or insulating-type thermally activated conduction. ${ }^{8}$ These hydrogen induced metal-semiconductor transitions greatly depend on the type of the rare earth and the substituting elements. ${ }^{8,9}$ It is reported that Ce-based com-

\footnotetext{
a) Author to whom correspondence should be addressed. Electronic mail: ramp@iitm.ac.in; Tel.: +91-44-22574862. FAX: +91-44-22570509.
}

pounds lead to stronger thermally activated conduction process upon hydrogenation than $\mathrm{HoCo}_{2}$. However, in the $\mathrm{Zr}$ substituted $\mathrm{HoCO}_{2}$ compounds, the hydrogen induced thermally activated conduction process drastically gets reduced with increasing $\mathrm{Zr}$ content. ${ }^{8}$

Therefore, it is of interest to investigate the structural and electrical transport properties of Laves phase rare earth and Co compounds and their hydrides with a mixture of LR and HR. In order to obtain a better understanding of the role played by hydrogen into driving the modifications of the structural, electrical, and magnetic behavior of these Laves phase compounds, here we present a detailed $\mathrm{x}$-ray diffraction and electrical resistivity studies in the $\mathrm{Ho}_{1-x} \mathrm{Mm}_{x} \mathrm{Co}_{2}$ $(x=0,0.1,0.2,0.3$, and 0.4 and $\mathrm{Mm}=$ mischmetal) alloys and their hydrides. Mischmetal is a cheap natural ore and contains the light rare earth metals: $50 \% \mathrm{Ce}, 35 \% \mathrm{La}, 8 \% \mathrm{Pr}, 5 \%$ $\mathrm{Nd}, 1.5 \%$ other rare earth elements and $0.5 \% \mathrm{Fe}$. Recently, studies on the hydrogen absorption and desorption properties of $\mathrm{Ho}_{1-x} \mathrm{Mm}_{x} \mathrm{Co}_{2}$ alloys ${ }^{18}$ using pressure-composition $(P-C)$ isotherms indicate the formation of two hydride phases, i.e., one intermetallic phase dissolving hydrogen (solid solution of the hydrogen in the metal, $\alpha$ phase) and another hydride phase ( $\beta$ phase), separated by an $(\alpha+\beta)$ two-phase region. Further, thermogravimetric (TG) analysis shows that the hydrides are in very stable nature at room temperature. In this 
TABLE I. The lattice parameters, unit cell volume expansion, and densities of alloys and their hydrides.

\begin{tabular}{|c|c|c|c|c|c|c|c|c|c|}
\hline \multirow[b]{3}{*}{$\begin{array}{l}\text { Alloy hydride } \\
\text { composition }\end{array}$} & \multicolumn{3}{|c|}{ Alloys } & \multicolumn{6}{|c|}{ Hydrides } \\
\hline & \multirow{2}{*}{$\begin{array}{l}\text { Lattice } \\
\text { Parameter } \\
(\AA)\end{array}$} & \multirow[b]{2}{*}{$\begin{array}{l}\text { Density } \\
(\mathrm{g} / \mathrm{cc})\end{array}$} & \multirow{2}{*}{$\begin{array}{l}\text { Volume } \\
\text { expansion, } \\
\Delta \mathrm{V} / \mathrm{V}_{(\mathrm{Mm}=0)} \%\end{array}$} & \multicolumn{2}{|l|}{ Two phase } & \multicolumn{2}{|l|}{ Hydride phase } & \multicolumn{2}{|c|}{$\begin{array}{l}\text { Volume expansion, } \\
\Delta \mathrm{V} / \mathrm{V}_{(y=0)} \%\end{array}$} \\
\hline & & & & $\begin{array}{l}\text { Lattice } \\
\text { parameter }(\AA)\end{array}$ & $\begin{array}{l}\text { Density } \\
(\mathrm{g} / \mathrm{cc})\end{array}$ & $\begin{array}{l}\text { Lattice } \\
\text { parameter }(\AA)\end{array}$ & $\begin{array}{l}\text { Density } \\
(\mathrm{g} / \mathrm{cc})\end{array}$ & Two phase & $\begin{array}{l}\text { Hydride } \\
\text { phase }\end{array}$ \\
\hline $\mathrm{HoCo}_{2}-\mathrm{H}_{y}$ & $7.1781(2)$ & 10.12 & $\cdots$ & $7.60(y=1.7)$ & 6.72 & $7.70(y=3.8)$ & 5.77 & 18.69 & 23.44 \\
\hline $\mathrm{Ho}_{0.9} \mathrm{Mm}_{0.1} \mathrm{Co}_{2}-\mathrm{H}_{y}$ & $7.1839(2)$ & 9.88 & 0.243 & $7.57(y=1.9)$ & 6.00 & $7.72(y=3.5)$ & 5.62 & 16.82 & 23.91 \\
\hline $\mathrm{Ho}_{0.8} \mathrm{Mm}_{0.2} \mathrm{Co}_{2}-\mathrm{H}_{y}$ & $7.1900(1)$ & 9.46 & 0.498 & $7.61(y=1.9)$ & 5.89 & $7.73(y=3.5)$ & 5.47 & 18.43 & 24.43 \\
\hline $\mathrm{Ho}_{0.7} \mathrm{Mm}_{0.3} \mathrm{Co}_{2}-\mathrm{H}_{y}$ & $7.1966(2)$ & 9.31 & 0.775 & $7.62(y=2.1)$ & 5.63 & $7.77(y=3.7)$ & 5.12 & 18.75 & 25.95 \\
\hline $\mathrm{Ho}_{0.6} \mathrm{Mm}_{0.4} \mathrm{Co}_{2}-\mathrm{H}_{y}$ & $7.2040(1)$ & 9.11 & 1.086 & $7.61(y=1.9)$ & 6.46 & $7.72(y=3.6)$ & 5.10 & 17.64 & 23.16 \\
\hline
\end{tabular}

paper we are concerned with crystalline hydrides of $\mathrm{Ho}_{1-x} \mathrm{Mm}_{x} \mathrm{Co}_{2}-\mathrm{H}_{y}$ with $0 \leqslant y \leqslant 3.6$. The coexistence of two hydride phases at lower hydrogen concentrations and the lattice expansion at higher $\mathrm{H}$ contents have been reported. The changes in magnetic transition and ordering temperature upon Mm concentration and hydrogen loadings have been discussed. Further, the effect of hydrogen interstitials on the transition from metallic state to thermally activated conducting state in these LR and HR mixture compounds has been discussed.

\section{EXPERIMENTAL DETAILS}

$\mathrm{Ho}_{1-x} \mathrm{Mm}_{x} \mathrm{Co}_{2}(x=0,0.1,0.2,0.3$, and 0.4) alloys were prepared by arc melting of stoichiometric amounts of constituent elements $\mathrm{Ho}, \mathrm{Mm}$, and Co with a purity better than 99.9\% under a protective argon atmosphere. $6 \mathrm{wt} \%$ excess of Ho and $\mathrm{Mm}$ were taken in order to prevent the formation of Co-rich phases. The ingots were melted several times to ensure homogeneity. Samples thus obtained were sealed in an evacuated quartz tube and homogenized at $1123 \mathrm{~K}$ for 4 days. Hydrogen absorption experiments were performed using the Sieverts-type apparatus. The ingot of annealed samples were preheated under high vacuum at $700 \mathrm{~K}$ for $2 \mathrm{~h}$ and then exposed to a controlled amount of hydrogen pressure, $0.001-1$ bar at $400 \mathrm{~K}$. The amount of $\mathrm{H}$ absorbed was determined volumetrically by monitoring the pressure changes in a calibrated volume. In order to get a homogeneous hydrogen distribution, the samples were annealed in a hydrogen atmosphere at $400 \mathrm{~K}$. The hydrogen desorption has been carried out by continuously evacuating the system and increasing the temperature up to $1000 \mathrm{~K}$. The structural and phase identification of alloys and their hydride samples have been carried out using powder x-ray diffraction (XRD). The powder XRD measurements were performed at room temperature using $X^{\prime}$ pert Pro, PANalytical diffractometer with $2 \theta$ values between $15^{\circ}$ and $90^{\circ}$, in steps of $0.05^{\circ}$ using $\mathrm{Cu} K \alpha$ radiation. The unit cell parameters were determined by a least-squares refinement method using silicon $(5 N)$ as an internal standard. The electrical resistivity measurements have been carried out using four-point contact technique on the circular disk shaped bulk $\mathrm{Ho}_{1-x} \mathrm{Mm}_{x} \mathrm{Co}_{2}$ alloys, buttons with about $8 \mathrm{~mm}$ diameter and 1-2 $\mathrm{mm}$ thickness, obtained by cutting the arc melted alloy using low speed diamond wheel saw and on samples made of compressed powders of its hydrides having the same dimensions. The densities of the bulk alloys and compressed hydride powders are listed in Table I. The voltage and current leads were attached to the samples using silver paint. The samples were mounted on an electrically insulating support and kept near to the cold head of a closed cycle refrigerator. The temperature was measured using a platinum resistance thermometer (PT100) in the range of $20-300 \mathrm{~K}$. The resistivity measurements down to $5 \mathrm{~K}$ have been carried out using a dip-stick helium cryostat, and the temperature has been measured using cernox sensor in the range of 5-30 K. The dc current through the sample was set in the range of $1 \times 10^{-1}-1 \times 10^{-3} \mathrm{~A}$ and the current was first applied in one direction and then reversed, in order to minimize the thermo-emf effects. Measurements at temperatures higher than $300 \mathrm{~K}$ could not be made due to the desorption of hydrogen from the hydride samples.

\section{RESULTS AND DISCUSSION}

\section{A. Crystal structure and lattice constant of alloys}

The XRD of homogenized $\mathrm{Ho}_{1-x} \mathrm{Mm}_{x} \mathrm{Co}_{2}(x=0,0.1,0.2$, 0.3 , and 0.4 ) alloys with Rietveld analysis shows ${ }^{19}$ that all the alloys crystallize in cubic Laves phase with the $\mathrm{MgCu}_{2}$-type structure (space group $F d \overline{3} m$ ). The lattice parameters $(a)$ at room temperature and the cell volume expansion upon Mm substitution are summarized in Table I. The lattice parameters increase linearly with increasing Mm concentration, which is ascribed to the larger radius of the $\mathrm{Mm}$ than that of Ho, since the mischmetal is an ore of lighter rare earths, which have larger radii than the heavier rare earth Ho.

\section{B. Hydrogen absorption and structural behavior of hydrides}

In order to understand the formation of different hydride phases and the contribution of each hydride phase to the structural property of $\mathrm{Ho}_{1-x} \mathrm{Mm}_{x} \mathrm{Co}_{2}$ alloys, the XRD of hydrogenated $\mathrm{Ho}_{1-x} \mathrm{Mm}_{x} \mathrm{Co}_{2}$ alloys with various hydrogen concentrations are investigated. For example, Fig. 1 shows the XRD patterns of the parent, the dehydrogenated (hydrogenated and then dehydrogenated), and hydrogenated $\mathrm{Ho}_{0.8} \mathrm{Mm}_{0.2} \mathrm{Co}_{2}$ alloys with different hydrogen concentrations in the range of $0-3.6 \mathrm{H} / \mathrm{f}$.u. at room temperature. The XRD pattern of the dehydrogenated sample resembles that of the parent alloy, without a shift of peak positions or appear- 


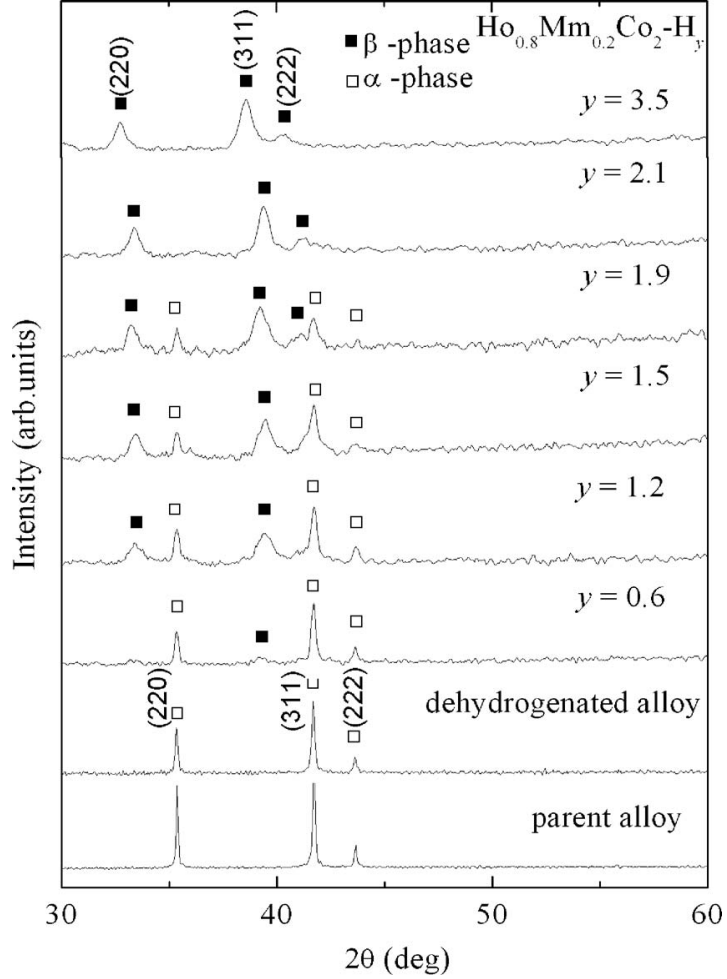

FIG. 1. Powder XRD patterns of $\mathrm{Ho}_{0.8} \mathrm{Mm}_{0.2} \mathrm{Co}_{2}-\mathrm{H}_{y}$, with hydrogen concentration range of $0-3.6 \mathrm{H} /$ f.u.

ance of the new peaks, except for a small decrease in the intensity. These two facts indicate that there is a slight decrease in grain size due to the plastic deformation. The large cell volume expansion upon hydrogenation and brittle nature of these alloys lead to fine hydride powders. The hydrides of $\mathrm{Ho}_{1-x} \mathrm{Mm}_{x} \mathrm{Co}_{2}$, with different concentrations maximum up to $y=3.6 \mathrm{H} / \mathrm{f}$.u., were prepared by controlling the sample temperature and pressure in the range of $0.001-1$ bar. A singlephase $\mathrm{C} 15 \mathrm{MgCu}_{2}$-type diffraction pattern is observed for $y$ $\leqslant 0.5$ and $y \geqslant 2$. In the concentration range $0.5 \leqslant y \leqslant 2$, all the diffraction lines split into two sets of identical lines, representing the coexistence of the two hydride phases. In accordance with the well-defined plateau in the $P-C$ isotherms of $\mathrm{Ho}_{1-x} \mathrm{Mm}_{x} \mathrm{Co}_{2}-\mathrm{H}_{y},{ }^{18} \mathrm{XRD}$, perturbed angular correlation (PAC) and Mossbauer measurements of isostructural Laves phase $R M_{2}-\mathrm{H}_{y}$ (Refs. 20-22) suggest that this region exhibits coexistence of two hydride phases. One phase (i.e., $\alpha$ phase) has a lattice parameter close to that of the unhydrogenated $\mathrm{Ho}_{1-x} \mathrm{Mm}_{x} \mathrm{Co}_{2}$ alloys and the other phase ( $\beta$ phase) corresponds to the hydride phase of $\mathrm{Ho}_{1-x} \mathrm{Mm}_{x} \mathrm{Co}_{2}$. The appearance of new set of diffraction lines with large shift towards the lower angle side represents large lattice expansion, due to the growth of the $\beta$ phase at the expense of the $\alpha$ phase. However, within the two-phase region, the relative change of amounts of the two phases changes, i.e., the ratio of amount of $\beta$ phase to $\alpha$ phase increases as observed by the change in the relative intensities of two identical sets of XRD lines (Fig. 1). Whereas in the $\beta$-phase region $(y \geqslant 2)$, the diffraction lines systematically shift further towards the smaller angles with increasing $y$, indicating increase in the lattice constant. The concentration dependence of the expansion in lattice constant and the unit cell volume of

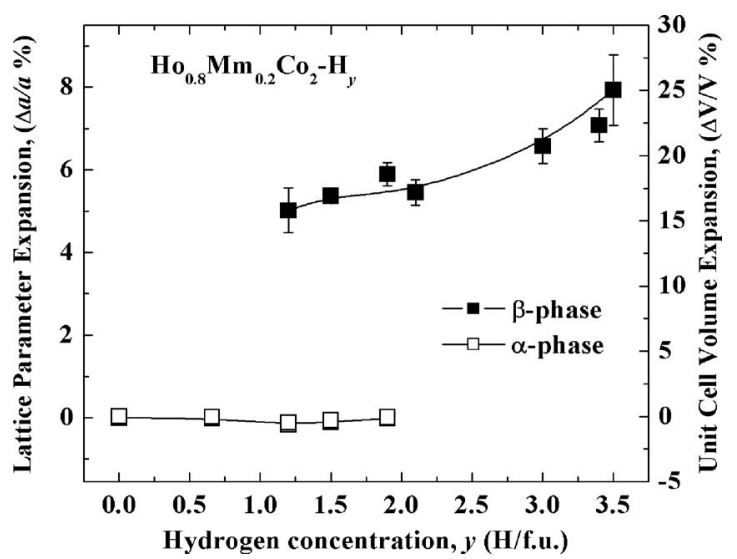

FIG. 2. Variation of lattice constant and corresponding unit cell volume of $\mathrm{Ho}_{0.8} \mathrm{Mm}_{0.2} \mathrm{Co}_{2}-\mathrm{H}_{y}$ upon increase in hydrogen concentration.

$\mathrm{Ho}_{0.8} \mathrm{Mm}_{0.2} \mathrm{Co}_{2}-\mathrm{H}_{y}$ are shown in Fig. 2. In the two-phase region, the absorbed hydrogen causes a negligible lattice expansion. In addition, the hydrogen concentration dependence of the lattice constant and the corresponding unit cell volume expansion of $\mathrm{Ho}_{1-x} \mathrm{Mm}_{x} \mathrm{Co}_{2}-\mathrm{H}_{y}$ in the two-phase and hydride phase region are summarized along with the lattice parameters of parent alloys in Table I. The lattice expansion in the $\beta$ phase is consistent with the typical volume increase of $2.9 \AA^{3}$ per hydrogen atom observed for different metalhydrogen systems. ${ }^{23}$ The present work shows that the formation of hydrides in $\mathrm{Ho}_{1-x} \mathrm{Mm}_{x} \mathrm{Co}_{2}$ does not lead to amorphization and all the hydrides retain their cubic $\mathrm{C} 15$ host lattice structure accompanied by substantial increase of the lattice constant.

\section{Temperature dependent electrical resistivity of $\mathrm{Ho}_{1-x} \mathrm{Mm}_{x} \mathrm{Co}_{2}$ alloys}

The temperature dependence of the electrical resistivity $\rho(T)$ of $\mathrm{Ho}_{1-x} \mathrm{Mm}_{x} \mathrm{Co}_{2}$ alloys for $x=0,0.1,0.2,0.3$, and 0.4 is shown in Fig. 3. The steplike behavior in the $\rho(T)$ plots indicates the magnetic transition temperature from paramagnetic state to magnetically ordered state. ${ }^{13,14}$ The magnetic ordering transition temperature $T_{C}$ has been determined from the maximum in $d \rho / d T$ vs $T$ plots. The observed $T_{C}$ of the parent $\mathrm{HoCO}_{2}$ is in agreement with earlier published data. $8,10,13,16$ The $\rho(T)$ curves of the $\mathrm{Ho}_{1-x} \mathrm{Mm}_{x} \mathrm{Co}_{2}$ compounds are characterized by nearly the same temperature dependence in the paramagnetic region. The total resistivity of $\mathrm{Ho}_{1-x} \mathrm{Mm}_{x} \mathrm{Co}_{2}$ includes several contributions and can be obtained from the Matthiessen's rule given by $\rho(T)=\rho_{0}$ $+\rho_{\mathrm{ph}}(T)+\rho_{\text {mag }}(T)$, where $\rho_{0}$ is the residual resistivity, $\rho_{\mathrm{ph}}$ is the phonon contribution, $\rho_{\text {mag }}\left[=\rho_{\text {sf }}(T)+\rho_{\text {spd }}(T)\right]$ is the magnetic contribution containing two spin-dependent parts, where $\rho_{\text {sf }}$ is the spin fluctuation resistivity due to the scattering of the conduction electrons by spin fluctuations within the Co $3 d$ band and $\rho_{\text {spd }}$ is the spin disorder contribution arising from the scattering of $R 4 f$ moments. $\rho_{\text {spd }}$ is independent of temperature above $T_{C}$, but it shows a $T^{2}$ dependence below $T_{C}{ }^{24}$ The phonon contribution to resistivity $\rho_{\mathrm{ph}}$ at low temperatures varies as $\rho_{\mathrm{ph}}=b T^{5}$. $^{14,24}$ The resistivity plots of $\mathrm{Ho}_{1-x} \mathrm{Mm}_{x} \mathrm{Co}_{2}$ below $T_{C}$ could be fitted well to the expression $\rho(T)=\rho_{0}+A T^{2}+B T^{5}$. The fit indicates that the $T^{2}$ term is 


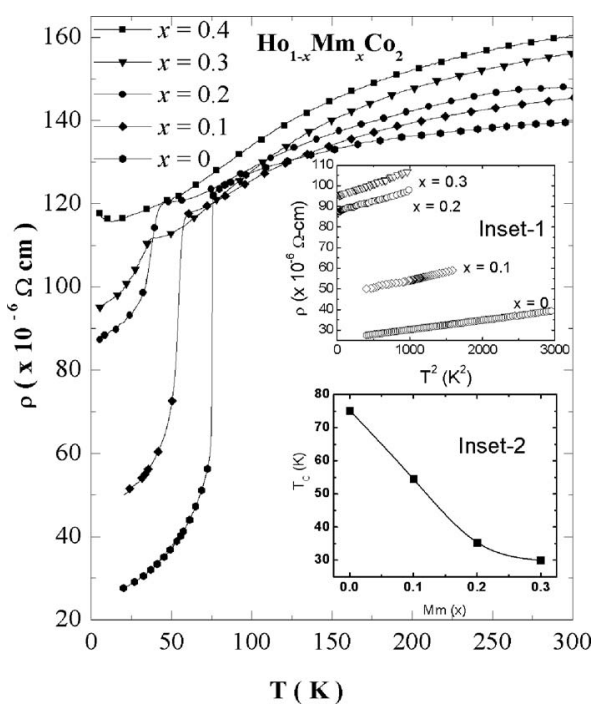

FIG. 3. Temperature dependent electrical resistivity of $\mathrm{Ho}_{1-x} \mathrm{Mm}_{x} \mathrm{Co}_{2}$ alloys for $x=0,0.1,0.2,0.3$, and 0.4 . Inset $1: T^{2}$ dependence of electrical resistivity below the magnetic transition temperature. Inset 2: variation of Curie temperature with $\mathrm{Mm}$ concentration, defined by the maximum in the $d \rho / d T$ vs $T$.

dominating. Thus inset 1 of Fig. 3 shows the $T^{2}$ dependence of resistivity well below $T_{C}$, which is attributed to the $\rho_{\text {spd. }}{ }^{24}$ A tendency for strong saturation in $\rho(T)$, observed above $T_{C}$ in all the cases, is a peculiar behavior pertaining to the $R \mathrm{Co}_{2}$ compounds. Such behavior is not seen in the isostructural $R \mathrm{TM}_{2}$ compounds with $\mathrm{TM}=\mathrm{Al}, \mathrm{Fe}$, or $\mathrm{Ni}$ and is attributed to the presence of spin fluctuations. ${ }^{14}$ The sharp drop in $\rho(T)$ plots at $T_{C}$ indicates the sudden appearance of the molecular field due to the spontaneous alignment of the $R$ moments. The molecular field induces magnetic moments on the Co sites and thus suppresses the spin fluctuations below $T_{C}{ }^{13,14}$ The slight increase in $\rho$ vs $T$ is observed when approaching $T_{C}$ from the paramagnetic temperature range. This can be ascribed to short-range order effects existing in both localized $4 f$ and itinerant $3 d$ sublattices, which enhances the spindensity fluctuations in the Co sublattice due to the $f$ - $d$ exchange coupling.

As can be seen, upon substituting $\mathrm{Mm}$ for Ho, the residual resistivity $\rho_{0}$ increases drastically and modifies the $\rho$ vs $T$ curves significantly in the low temperature region. Unlike the dominant $T^{2}$ dependence of the low temperature resistivity, pronounced minimum appears in the $\rho(T)$ curve for $x=0.4$. Usually, minima in the temperature dependence of $\rho(T)$ are associated with freezing of the magnetic moments of ferro/ferrimagnetic clusters in random directions. ${ }^{25-27} \mathrm{An}$ increasing extent of spin fluctuations and a probable instability of the $R$ moments are also evidenced by low temperature specific heat measurements in the dilute $R$ concentrations. ${ }^{28}$ The variation of $T_{C}$ upon $\mathrm{Mm}$ substitution is shown in inset 2 of Fig. 3. The strong nonlinear decrease of $T_{C}$ is attributed to the decreasing $f-d$ exchange interactions with increasing Mm content. ${ }^{11}$ The effect of dilution of the magnetic $R$ ions and influence on the resistivity have been investigated for several pseudobinary $(R, Y) \mathrm{Co}_{2}$ and $\mathrm{LR}_{1-x} \mathrm{HR}_{x} \mathrm{Co}_{2}$ compounds. ${ }^{8,13,14,29-32}$ These studies suggest the weakening of magnetic interactions due to the dilution of the $R$ moment by a non- or less magnetic element, thereby decreasing the molecular field. Therefore, the observed decrease in the $T_{C}$ is attributed to the weak magnetic interactions due to the $\mathrm{Mm}$ substitution.

\section{Temperature dependence of electrical resistivity of $\mathrm{Ho}_{1-x} \mathrm{Mm}_{x} \mathrm{Co}_{2}$ hydrides}

Figures 4(a)-4(e) display the temperature dependence of the reduced representation of electrical resistivity $\rho(T)$ for the $\mathrm{Ho}_{1-x} \mathrm{Mm}_{x} \mathrm{Co}_{2}-\mathrm{H}_{y}$ samples in the temperature range of $20-300 \mathrm{~K}$. Each figure contains parent alloy, dehydrogenated (hydrogenated and then dehydrogenated) and a series of hydrogenated $\mathrm{Ho}_{1-x} \mathrm{Mm}_{x} \mathrm{Co}_{2}$ samples at different hydrogen concentrations in the range of $0 \leqslant y \leqslant 3$.6. The resistivity of dehydrogenated samples shows a smooth change in magnetic transitions and a large increase in the reduced residual resistivity, unlike the sudden changes observed in the parent alloys. It is reported that the dehydrogenated samples of $\mathrm{PrCo}_{2}$ and $\mathrm{NdCo}_{2}$ fail to show the sudden change in resistivity as observed in parent $\mathrm{PrCo}_{2}$ and $\mathrm{NdCo}_{2}$. ${ }^{1}$ Therefore, the $\rho(T)$ plots indicate that the dehydrogenated $\mathrm{Ho}_{1-x} \mathrm{Mm}_{x} \mathrm{Co}_{2}$ samples exhibit weak magnetic interactions.

At lower hydrogen concentrations up to about $y \leqslant 2$ in these alloy hydrides, the magnetic transition temperature gradually disappears with increasing hydrogen concentrations, as observed by the smoothening of $\rho(T)$ plots from the steplike behavior of unhydrogenated alloys. There is a substantial increase in lattice parameters confirmed from the XRD studies of hydrogenated $\mathrm{Ho}_{1-x} \mathrm{Mm}_{x} \mathrm{Co}_{2}$ samples. In addition, the previously reported XRD, Mossbauer, magnetization, ac susceptibility, and electrical resistivity studies on the $R-\mathrm{H}_{y}$ and Laves phase $R \mathrm{Co}_{2}-\mathrm{H}_{y}$ type systems $\mathrm{s}^{1,2,5,8,16,17,33-36}$ conclude that with increasing hydrogen concentration, there is a large increase in lattice parameters, substantial reduction of conduction electron density, lack of saturation in magnetization, a decrease in the Co moment and fanning of the rare earth moments, and gradual reduction or disappearance of magnetic transition temperature. Therefore the large lattice expansion and decrease in the number of conduction electrons due to charge transfer between hydrogen and the $3 d$ band in hydrogenated $\mathrm{Ho}_{1-x} \mathrm{Mm}_{x} \mathrm{Co}_{2}$ lead to a decrease of the strength of various exchange interactions of the local moments mediated by the conduction electrons. This decrease modifies the magnitudes of the $3 d$ moments and weakening of $R-$ Co interactions, which will affect the magnetic scattering contribution to the electrical resistivity significantly. ${ }^{8,16}$ Consequently, the type of magnetic order changes with increasing hydrogen concentrations and the broadening of transition at $T_{C}$ has been observed in the hydrides. As $y$ increases, the magnitude of the $\rho / \rho_{(300 \mathrm{~K})}$ values at $20 \mathrm{~K}$ increases considerably due to the decrease in the conduction electron density.

However, $\rho$ vs $T$ plots show different behaviors at intermediate $\mathrm{H}$ concentrations and they exhibit several general characteristic features as a function of $T$. As a typical example, we consider the results for the $\mathrm{Ho}_{0.9} \mathrm{Mm}_{0.1} \mathrm{Co}_{2}-\mathrm{H}_{y}$ sample with $y=2$ [Fig. 5(a)]. With respect to the sign of the temperature derivative of $\rho$ (i.e., $d \rho / d T$ ), three conduction 

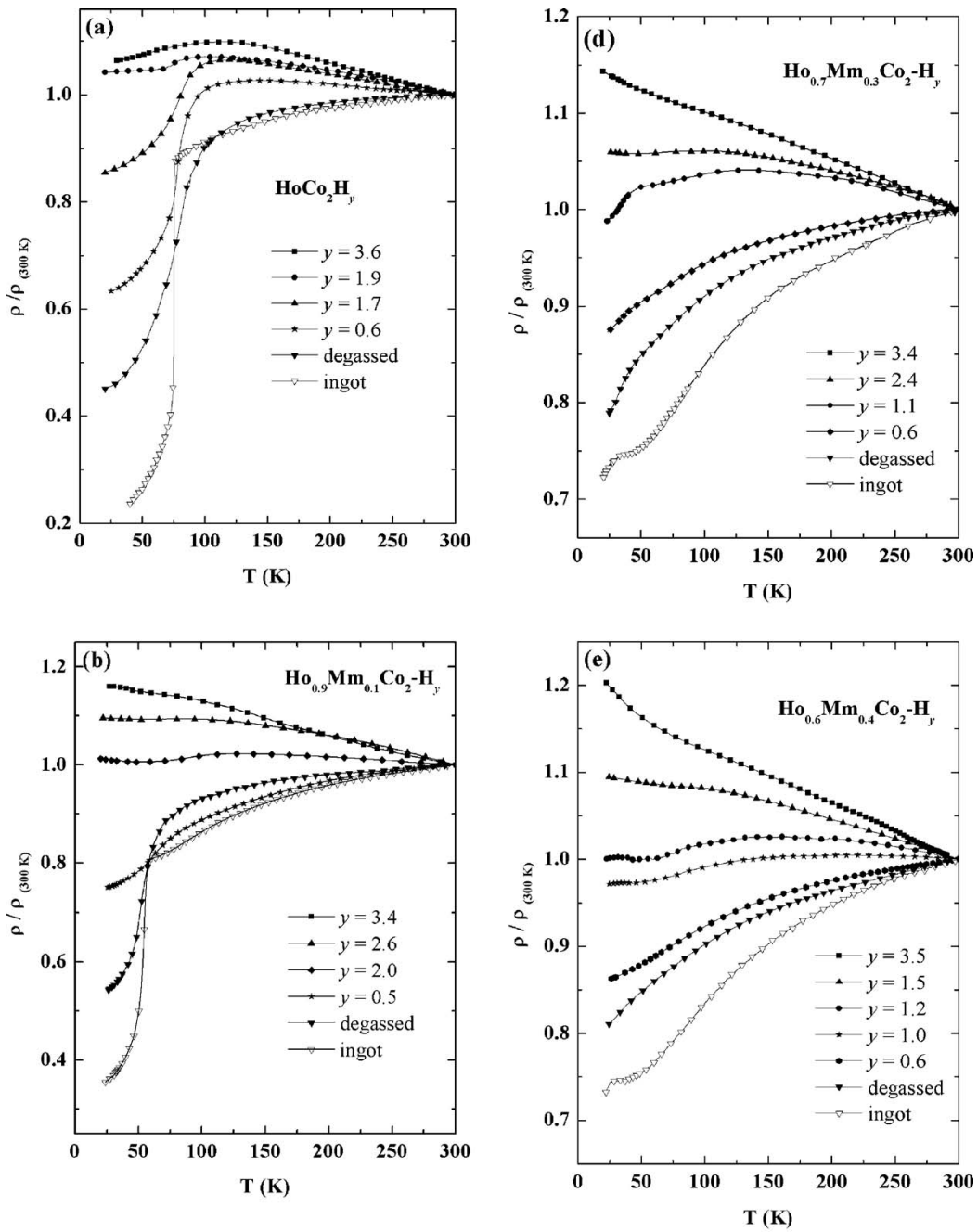

FIG. 4. [(a)-(e)] Temperature dependent normalized representation of electrical resistivity of $\mathrm{Ho}_{1-x} \mathrm{Mm}_{x} \mathrm{Co}_{2}-\mathrm{H}_{y}(x=0,0.1,0.2,0.3$, and 0.4$)$ for unhydrogenated, dehydrogenated, and hydrogenated at different hydrogen concentrations $(y)$ in the range of 0-3.6 H/f.u.

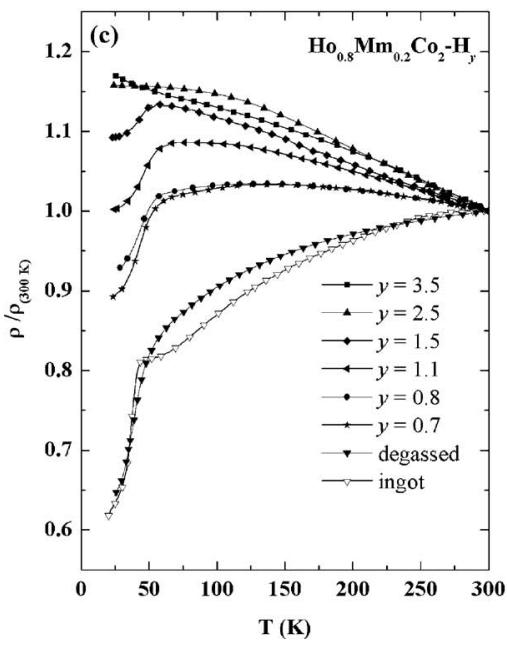

regimes are distinguished. At high temperatures, in regime (III), the electrical resistivity increases with decreasing temperature, which makes $d \rho / d T<0$. This temperature activated process is possible down to $\sim 130 \mathrm{~K}$. At intermediate temperatures, in regime (II), $\rho(T)$ exhibits metalliclike behavior with a positive $d \rho / d T$. This holds down to $T_{\min } \sim 50 \mathrm{~K}$, where the electrical resistivity goes to a minimum. Then resistivity again increases below about $50 \mathrm{~K}$ in regime (I) with $d \rho / d T<0$. A similar behavior is observed for the rare earth hydride systems ${ }^{37-41}$ with resistivity maxima appearing around $200-260 \mathrm{~K}$. It is attributed that the hydrogen sublattice forms an ordered superlattice at the lowest temperatures and at higher temperatures, this superlattice is destroyed due to the rapid motion of the hydrogen atoms. This disorderinduced mechanism causes a transition to region $d \rho / d T<0$, which is a characteristic of a semiconductor or an insulator. The temperature variation of resistivity in semiconductors is usually described by various functional forms, such as thermal activation and variable range hopping; i.e., $\rho$ $\approx \exp \left(-T_{0} / T\right)^{p}$ where $p=1$ represents a simple thermally ac- 

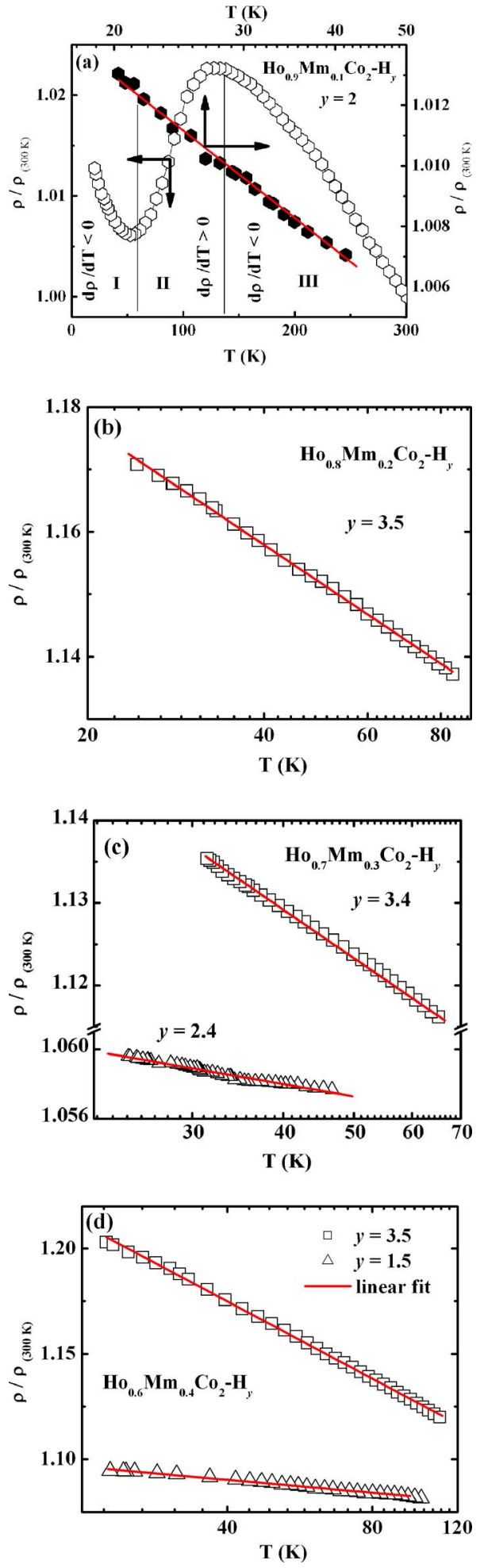

FIG. 5. [(a)-(d)] The straight line behavior of $\rho / \rho_{(300 \mathrm{~K})} \mathrm{vs} \ln T$ plots for $\mathrm{Ho}_{1-x} \mathrm{Mm}_{x} \mathrm{Co}_{2}-\mathrm{H}_{y}(x=0,0.1,0.2,0.3$, and 0.4$)$ at the low temperature region.

tivated behavior and $p=1 / 3$ is consistent with variable-range hopping. ${ }^{42}$ However, in the low temperature regime (I), below $50 \mathrm{~K}$, we find a logarithmic $T$ dependence of $\rho(T)$ [Fig. 5(a)]. This could be due to Kondo scattering of the free electrons by the localized magnetic clusters. ${ }^{1,43-49}$

For the analysis of $\rho(T)$ plots of $\mathrm{Ho}_{1-x} \mathrm{Mm}_{x} \mathrm{Co}_{2}-\mathrm{H}_{y}$ at intermediate and higher hydrogen concentrations we use the following expression:

$$
\rho(T)=\rho_{0}-\rho_{1} \ln T+\rho_{2} T^{\alpha} .
$$

The Kondo term (i.e., $-\rho_{1} \ln T$ ) is important at low temperatures. The power-law term $\rho_{2} T^{\alpha}$ is the contribution to $\rho$ from electron-phonon or electron-electron scattering processes. For electron-phonon scattering, the power-law term scales as $T(\alpha=1)$ at high temperatures and as $T^{5}(\alpha=5)$ at low temperatures. ${ }^{50}$ Electron-electron scattering leads to a $T^{2}$ contribution to $\rho .^{51}$ At lower temperature, the $\rho(T)$ plots are well described by $\rho(T)=\rho_{0}-\rho_{1} \ln T$, characteristic of spinglass or Kondo effect, and this straight line behavior of $\rho / \rho_{(300 \mathrm{~K})}$ vs $\ln T$ plots is shown in Figs. 5(a)-5(d). Figures 6(a)-6(e) shows the remaining electrical resistivity $\rho(T)$ plots for $\mathrm{Ho}_{1-x} \mathrm{Mm}_{x} \mathrm{Co}_{2}-\mathrm{H}_{y}$ to exhibit a $T^{\alpha}$ power-law dependence over a wide temperature range. A fit of $\rho(T) \sim T^{\alpha}$ yields $\alpha=1.8 \pm 0.3$, therefore $\rho(T)$ is dominated by electronelectron scattering effects.

The appearance of minima in the two-phase region upon lowering temperature can be interpreted with combined resistivity behaviors of the $\alpha$ phase and $\beta$ phase. Probably, in the two-phase region, there may be a percolation problem due to the mixture of these two phases. Further, it is attributed to the temperature dependence of miscibility gap (plateau region) of hydrides, which may broaden upon lowering temperature. Increasing $\mathrm{H}$ concentration, it can be seen that the resistivity minima gets considerably reduced and completely vanishes in $\mathrm{Ho}_{0.9} \mathrm{Mm}_{0.1} \mathrm{Co}_{2}-\mathrm{H}_{2.6}$ and $\mathrm{Ho}_{0.9} \mathrm{Mm}_{0.1} \mathrm{Co}_{2}-\mathrm{H}_{3.4}$, respectively, [Fig. 4(b)]. At higher hydrogen concentrations all the $\rho(T)$ plots of $\mathrm{Ho}_{1-x} \mathrm{Mm}_{x} \mathrm{Co}_{2}-\mathrm{H}_{y}$ represent the thermally activated conduction mechanism throughout the measured temperature region. One must note that this minimum tends to disappear with increasing $\mathrm{H}$ concentration, where the system goes from a two-phase region to a single hydride phase region. It does not mean that there is no Kondo effect, since the contribution to the resistivity from the $\alpha$ phase decreases with increasing $\mathrm{H}$ concentration. In the hydride phase even though there is no minima in $\rho(T)$ plots at the lower temperature region, the very well defined Kondo behavior can be seen in Figs. 5(a)-5(d) in a wide temperature range. Further, it can be seen from Figs. 5(c) and 5(d) that, when approaching the pure hydride phase with increasing $\mathrm{H}$ concentration, the slope of $d \rho(T) / d \ln T$ at lower temperatures increases. This strong increase in the slope of the resistivity with increasing $\mathrm{H}$ concentration reveals enhanced Kondo scattering of the free electrons by the localized magnetic clusters. This may be attributed to the increasing localized magnetic clusters. Thus, $\rho(T)$ plots in the $\beta$-phase region suggests full suppression of the long-range magnetic ordering and the presence of either a weak magnetic interaction or the development of paramagnetic or antiferromagnetic clusters, which indicate the onset of incommensurate order or of magnetic fluctuations.

\section{CONCLUSION}

The structural analysis of $\mathrm{Ho}_{1-x} \mathrm{Mm}_{x} \mathrm{Co}_{2}-\mathrm{H}_{y}(x=0,0.1$, $0.2,0.3$, and 0.4$)$ in the hydrogen concentration range of $0-3.6 \mathrm{H} /$ f.u. reveals the existence of different hydride phases, which are distinguished by difference in the lattice constants. $\mathrm{Ho}_{1-x} \mathrm{Mm}_{x} \mathrm{Co}_{2}$ hydrides retain their crystalline na- 

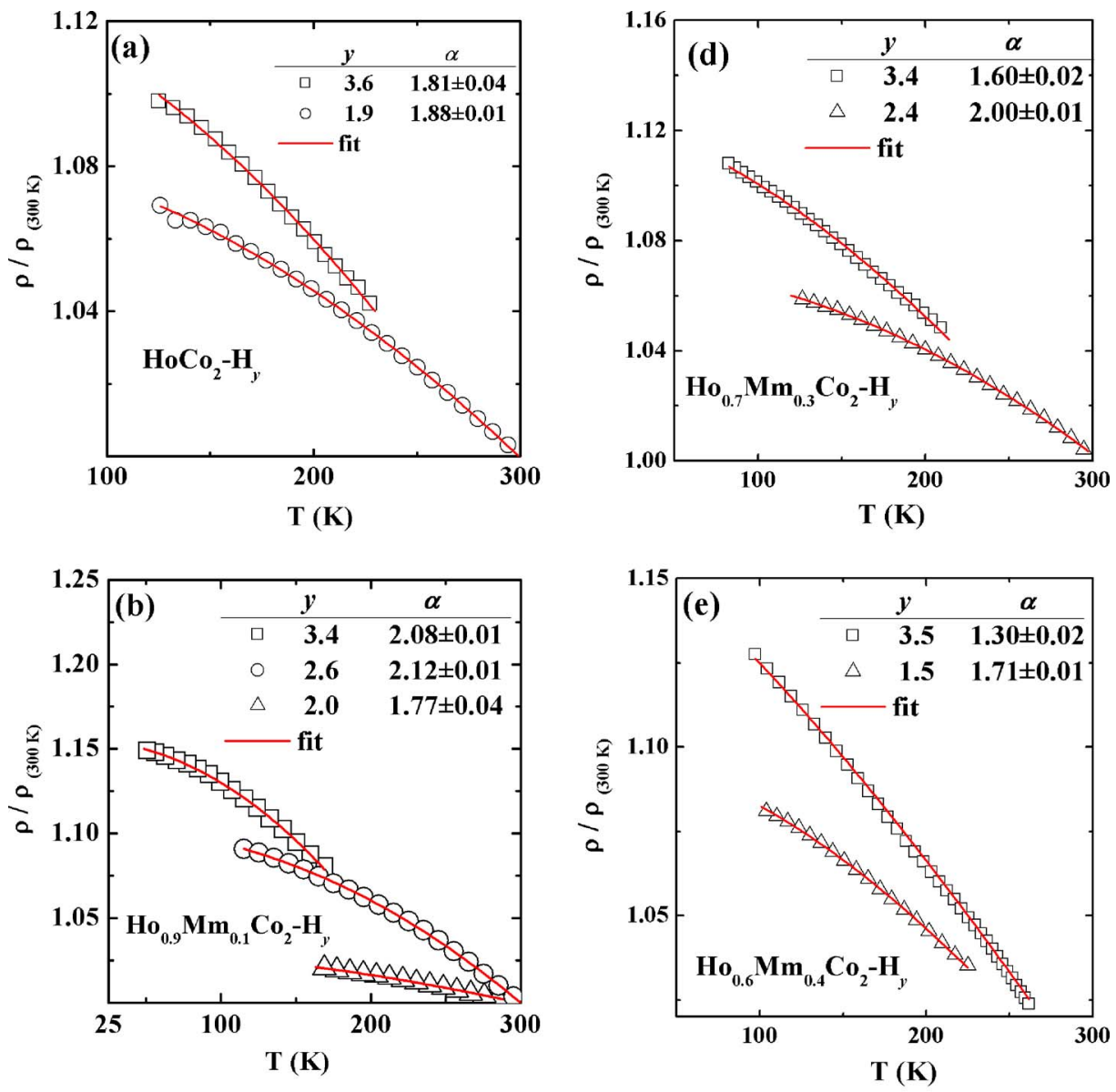

FIG. 6. [(a)-(e)] $\rho / \rho_{(300 \mathrm{~K})}$ vs $T$ plots for $\mathrm{Ho}_{1-x} \mathrm{Mm}_{x} \mathrm{Co}_{2}-\mathrm{H}_{y}(x=0,0.1,0.2$, 0.3 , and 0.4 ) at higher temperatures; the solid line represents the fit of power-law dependence $\rho(T) \approx \rho_{2} T^{\alpha}$.

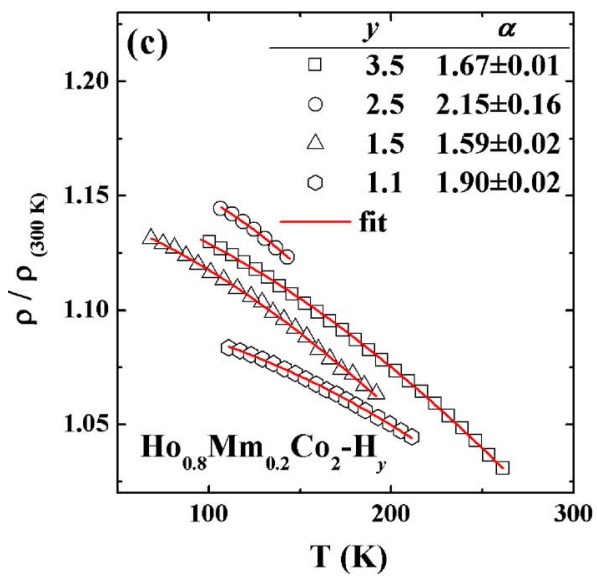

ture with $\mathrm{C} 15$ Laves structure without passing through the hydrogen-induced amorphization. The pronounced curvature in $\rho(T)$ plots at elevated temperatures is due to the spin fluctuation scattering of the conduction electrons. The substitution of $\mathrm{Mm}$ for Ho in $\mathrm{Ho}_{1-x} \mathrm{Mm}_{x} \mathrm{Co}_{2}$ decreases the exchange field acting on the $d$-electron subsystem and thus decreases the magnetic ordering transition temperature. The resistivity studies on the hydrogenated samples reveal a successive suppression of the Curie temperature and thermally activated conduction; the system goes from an ordered magnetic state to a Kondo type behavior. These findings are attributed to the large lattice expansion upon hydrogenation and reduction in the conduction electron density. The temperature dependence of the electrical resistivity at higher hydrogen concentrations is well described by $\rho(T)=\rho_{0}-\rho_{1} \ln T+\rho_{2} T^{\alpha}$, with $\alpha$ $=1.8 \pm 0.3$. The $\ln T$ dependence at lower temperatures is due to Kondo effect and the higher temperature region is dominated by electron-electron scattering effects.

\section{ACKNOWLEDGMENTS}

The authors acknowledge to the DRDO and DST for supporting this work. One of the authors (G. Srinivas) is grateful to IIT Madras for financial support.

${ }^{1}$ G. Alefeld and J. Volkl, Hydrogen in Metals-I, Topics in Applied Physics (Springer, Berlin, 1978), Vol. 28.

${ }^{2}$ L. Schlapbach, Hydrogen in Intermetallic Compounds-I, Topics in Applied Physics Vol. 63 (Springer, Berlin, 1988). 
${ }^{3}$ V. Paul-Boncour, J. Alloys Compd. 367, 185 (2004).

${ }^{4}$ F. Pourarian, Physica B 321, 18 (2002).

${ }^{5}$ P. Cadavez-Peres, I. N. Goncharenko, and I. Mirebeau, Phys. Rev. B 64, 094419 (2001).

${ }^{6}$ I. N. Goncharenko, P. Cadavez-Peres, I. Mirebeau, O. L. Makarova, T. Le Bihan, and M. Mezouar, Phys. Rev. B 68, 214418 (2003).

${ }^{7}$ S. Annapoorni, G. Markandeyulu, and K. V. S. Rama Rao, J. Phys. Soc. Jpn. 59, 3014 (1990).

${ }^{8}$ R. Ramesh and K. V. S. Rama Rao, J. Appl. Phys. 76, 3556 (1994).

${ }^{9}$ P. Raj, P. Suryanarayana, A. Sathyamoorthy, K. Shashikala, K. V. Gopalakrishnan, and R. M. Iyer, J. Alloys Compd. 179, 99 (1992).

${ }^{10}$ E. Gratz, R. Resel, A. T. Burkov, E. Bauer, A. S. Markosyan, and A. Galatanu, J. Phys.: Condens. Matter 7, 6687 (1995).

${ }^{11}$ E. Gratz et al., J. Phys.: Condens. Matter 7, 597 (1995).

${ }^{12}$ E. Gratz and A. S. Markosyan, J. Phys.: Condens. Matter 13, R385 (2001).

${ }^{13}$ N. H. Duc and P. E. Brommer, Handbook of Magnetic Materials, edited by K. H. J. Buschow (Elsevier, Amsterdam, 1999), Vol. 12, p. 259.

${ }^{14}$ J. M. Fournier and E. Gratz, Handbook on the Physics and Chemistry of Rare Earths, edited by K. A. Gschneidner, Jr., L. Eyring, G. H. Lander, and G. R. Choppin (Elsevier, Amsterdam, 1993), Vol. 17, p. 409.

${ }^{15}$ A. Slebarski, M. Matlak, and M. Hafez, J. Alloys Compd. 203, 35 (1994).

${ }^{16}$ F. Pourarian, W. E. Wallace, and S. K. Malik, J. Magn. Magn. Mater. 25, 299 (1982).

${ }^{17}$ R. Ramesh, M. S. Ramachandra Rao, and K. V. S. Rama Rao, J. Appl. Phys. 77, 2090 (1995).

${ }^{18}$ G. Srinivas, V. Sankaranarayanan, and S. Ramaprabhu, Int. J. Hydrogen Energy 32, 2480 (2007).

${ }^{19}$ G. Srinivas, V. Sankaranarayanan, and S. Ramaprabhu, J. Phys. D 40 1183 (2007).

${ }^{20}$ T. D. Saxce, Y. Berthier, and D. Fruchart, J. Less-Common Met. 107, 35 (1985).

${ }^{21}$ J. Zukrowski, M. Strecker, G. Wortmann, J. Przewoznik, and K. Krop, J. Alloys Compd. 261, 47 (1997).

${ }^{22}$ S. M. Van Eek and M. Forker, Phys. Rev. B 65, 174307 (2002).

${ }^{23}$ D. G. Westlake, J. Less-Common Met. 90, 251 (1983).

${ }^{24}$ T. Nakama, K. Shintani, K. Yagasaki, A. T. Burkov, and Y. Uwatoko, Phys. Rev. B 60, 511 (1999).

${ }^{25}$ E. Gratz, N. Pillmayr, E. Bauer, and G. Hilschen, J. Magn. Magn. Mater 70, 159 (1987).

${ }^{26}$ E. Gratz, E. Bauer, V. Sechorvsky, and J. Chmist, J. Magn. Magn. Mater. 54-57, 517 (1986).
${ }^{27}$ N. V. Baranov, A. A. Yermakov, and A. Podlesnyak, J. Phys.: Condens. Matter 15, 5371 (2003).

${ }^{28}$ N. Pillmayr, C. Schmitzer, E. Gratz, G. Hilscher, and V. Sechovsky, J. Magn. Magn. Mater. 70, 162 (1987).

${ }^{29}$ A. Podlesnyak, Th. Strassle, J. Schefer, A. Furrer, A. Mirmelstein, A. Pirogov, P. Markin, and N. Baranov, Phys. Rev. B 66, 012409 (2002).

${ }^{30}$ Z. W. Ouyang, G. H. Rao, H. F. Yang, W. F. Liu, G. Y. Liu, X. M. Feng, and J. K. Liang, J. Phys.: Condens. Matter 15, 5599 (2003).

${ }^{31}$ E. Gratz, R. Hauser, I. S. Dubenko, and A. S. Markosyan, J. Phys.: Condens. Matter 8, 11093 (1996).

${ }^{32}$ Z. W. Ouyang, G. H. Rao, H. F. Yang, W. F. Liu, and J. K. Liang, Appl. Phys. Lett. 81, 97 (2002).

${ }^{33}$ B. D. Dunlap, G. K. Shenoy, J. M. Friedt, P. J. Viccaro, D. Niarchos, H. Kierstead, A. T. Aldred, and D. G. Westlake, J. Appl. Phys. 50, 7682 (1979).

${ }^{34}$ R. L. Cohen, K. W. West, F. Oliver, and K. H. J. Buschow, Phys. Rev. B 21, 941 (1980)

${ }^{35}$ F. Pourarian, W. E. Wallace, and S. K. Malik, J. Less-Common Met. 83, 95 (1982).

${ }^{36}$ X. G. Li, A. Chiba, S. Takahashi, K. Aoki, and T. Masumoto, Intermetallics 7, 207 (1999).

${ }^{37}$ G. G. Libowitz, J. G. Pack, and W. P. Binnie, Phys. Rev. B 6, 4540 (1972).

${ }^{38}$ G. G. Libowitz, Ber. Bunsenges. Phys. Chem. 76, 837 (1972).

${ }^{39}$ J. Shinar, B. Dehner, B. J. Beaudry, and D. T. Peterson, Phys. Rev. B 37, 2066 (1988).

${ }^{40}$ P. Vajda, J. N. Daou, and J. P. Burger, Phys. Rev. B 40, 500 (1989).

${ }^{41}$ P. Vajda, J. N. Daou, and J. P. Burger, J. Less-Common Met. 172-174, 271 (1991).

${ }^{42}$ N. F. Mott, Metal-Insulator Transitions (Taylor \& Francis, London, 1990).

${ }^{43}$ J. Kondo, Prog. Theor. Phys. 32, 37 (1964).

${ }^{44}$ G. Gruner and A. Zawadowski, Rep. Prog. Phys. 37, 1497 (1974).

${ }^{45}$ J. F. DiTusa, K. Friemelt, E. Bucher, G. Aeppli, and A. P. Ramirez, Phys. Rev. Lett. 78, 2831 (1997).

${ }^{46}$ J. N. Huiberts, R. Griessen, R. J. Wijngaarden, M. Kremers, and C. Van Haesendonck, Phys. Rev. Lett. 79, 3724 (1997).

${ }^{47}$ S. Enache, W. Lohstroh, and R. Griessen, Phys. Rev. B 69, 115326 (2004).

${ }^{48}$ J. P. Burger, P. Vajda, and J. N. Daou, J. Phys. Chem. Solids 52, 779 (1991).

${ }^{49}$ J. A. Mydosh, Phys. Rev. Lett. 33, 1562 (1974).

${ }^{50}$ C. Kittel, Introduction to Solid State Physics (Wiley, New York, 1996).

${ }^{51}$ W. G. Baber, Proc. R. Soc. London, Ser. A 158, 383 (1973). 